\title{
Role of Low Level Laser Therapy (LLLT) on Myofascial Trigger Point in and around Neck - A Review
}

\author{
Dheeraj Lamba* \\ Department of Orthopaedic Physiotherapy, Associate Professor, Faculty of \\ Physiotherapy, Institute of Health, Jimma University, Jimma, Ethiopia \\ *Corresponding Author: Dr. Dheeraj Lamba, Department of Orthopaedic \\ Physiotherapy, Associate Professor, Faculty of Physiotherapy, Institute of \\ Health, Jimma University, Jimma, Ethiopia.
}

\section{Received: March 03, 2020}

Published: March 12, 2020

(C) All rights are reserved by Dheeraj Lamba.

\begin{abstract}
Myofascial trigger point (MTP) is a characteristic of myofascial pain syndrome (MPS) which is the most common muscle pain disorder. MPS is pain arising from one or more trigger points (TP) which are hyperirritable spots in skeletal muscle that are associated with hypersensitive palpable nodule in taut bands. There are lots of perpetuating factors for MTPs which can be divided under many heads like poor posture, muscle injury, nutritional inadequacies, metabolic or endocrine disorders, psychological factors, chronic injury, impaired sleep radiculopathy allergies and chronic visceral diseases. The major goal of any therapy is to relieve pain and increase functional ability. Currently used therapy includes various methods out of which low level laser therapy (LLLT) is one of the most common. LLLT can produce pain relief by one or a combination of these mechanisms - collagen proliferation, anti-inflammatory effect, circulation enhancement, and peripheral nerve stimulation. There has been a contrary evidence for the significant role of LLLT in musculoskeletal disorders, therefore it is up to the physical therapist to use LLLT in the clinical setup under available evidence based protocols for the treatment of musculoskeletal disorders particularly myofascial trigger points in and around neck.

Keywords: Myofascial Trigger Point (MTP); Myofascial Pain Syndrome (MPS), Low Level Laser Therapy (LLLT); Non-steroidal Anti-Inflammatory Drugs (NSAID); Gallium Aluminum Arsenide (Ga-As-Al); Helium Neon (He-Ne)
\end{abstract}

\section{Introduction}

Myofascial trigger point (MTP) is a characteristic of myofascial pain syndrome (MPS) which is the most common muscle pain disorder [7]. MPS is pain arising from one or more trigger points (TP) which are hyperirritable spots in skeletal muscle that are associated with hypersensitive palpable nodule in taut bands. There are lot of perpetuating factors for it like postural, mechanical, environmental stresses, emotional stresses and external compression. Trigger points can arise in virtually any muscle group however the trapezius muscle appears to be the most frequently cited in clinical settings [12]. Four muscles trapezius, levator scapulae, infraspinatus and scalenus accounts for $84.7 \%$ of TP. Out of these muscles, trapezius account for $34.7 \%$ and levator scapulae constitute $19.7 \%$ of TP. Common treatments consist of drugs, non-steroidal antiinflammatory drugs (NSAID) [6] and epidural injection. Various physical modalities include intermittent cold and stretch, thermotherapy, massage therapy, post isometric relaxation, dry needling, trigger point injections, ischemic compression, TENS, ultrasound and Low level laser therapy (LLLT).

\section{Pathophysiologic development of trigger points}

Trigger points occur in any skeletal muscle, most frequently in head, neck, shoulders and lower back. The referral pattern or zone of reference of each TP is consistent among different persons [8].

\section{Motor end plate}

Finding in pathology of myofascial pain reveals that there is an increase in release of acetylcholine (ACh) which is considered as an abnormal motor end plate activity [2,5]. This abnormality is considered to be the primary dysfunction in the integrated hypothesis proposed by Simon., et al. which postulates a positive feedback loop.

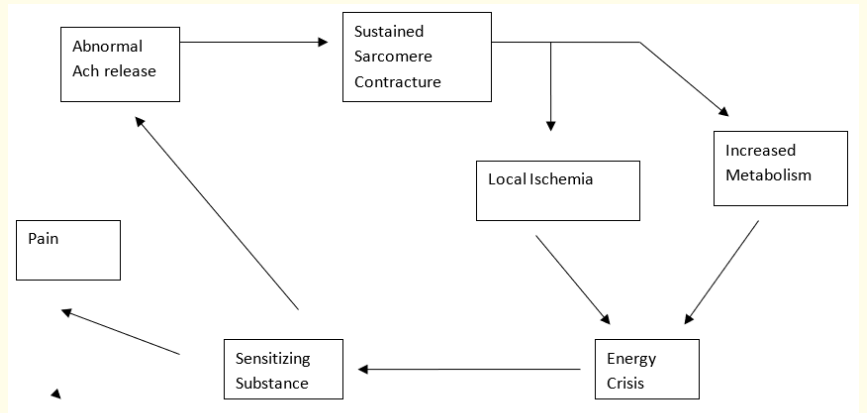

Figure 1: Positive feedback cycle.

\section{Muscle fibre}

It is hypothesized that increased Ach release could result in sustained depolarization of post functional membrane of the muscle 
fibre and produce sustained sarcomere shortening. This leads to local ischemia and increased metabolism of local fibres, which results in production of sensitizing substances such as PG, bradykinis, serotonins etc. [2].

\section{Other perpetuating factors}

Perpetuating factors for trigger point pain are mechanical stress such as poor posture or muscle injury, nutritional inadequacies, metabolic or endocrine disorders, psychological factors, chronic injury, impaired sleep radiculopathy allergies and chronic visceral diseases $[3,7,10]$.

\section{Activation of trigger point in muscle}

Overused or stress of the muscle containing Trigger point leads to activation of myofascial trigger point and in turn produces pain $[2,3,5,7]$.

\section{Central mechanism (Spinal and supraspinal)}

At the level of the central nervous system, spinal neuroplastic changes occur in the second order neuron pool of dorsal horn due to persistent pain. These changes lead to long lasting increase in excitability of nocioceptive pathways. Central sensitization result in characterized by increase excitability of neurons [2,28]. Hong., et al. [7] demonstrated that (LTR) depends mainly on CNS with a possible minor degree of local transmission.

\section{Posture}

Postural factors have been identified as a key contributing factor to myofascial pain syndrome (MPS) by numerous authors $[3,16]$. This includes static postural problems such as unilateral short leg, small hemi pelvis and scoliosis or functional postural habits [20]. Upper Trapezius function of neck stabilization is commonly over loaded by the tilting of the shoulder girdle axis due to a short leg or short hemi pelvis. The short leg tilts the pelvis laterally which bows into functional scioliotic curve and in turn tilts the shoulder, causing one to sag. Anette [21] reported $15.2 \%$ prevalence of myofascial pain syndrome in sewing machine operators. This is due to their static and poor posture they use for sewing. In a study of postural problems in 164 head and neck myofascial pain patients, Friction and associates [8] found poor sitting posture in $96 \%$, forward head in $84.7 \%$, rounded shoulders in $82.5 \%$, lower tongue position in $67.7 \%$, abnormal lordosis in $46.3 \%$, scoliosis in $15.9 \%$ and leg length discrepancy in $14 \%$. Simon's., et al. [1] attributed these due to constant tension and micro trauma due to postural habits, both in everyday living and in work place.

\section{Other factors}

- Nutritional Inadequacies: Low level of Vitamin B, B6, B12, Folic Acid, Vitamin C and Iron. Levine and Hartzell pointed out that Vitamin $\mathrm{C}$ is an essential for the synthesis of Norepinephrine and serotonin both important in central modulation of pain transmission [1].
- Metabolic and Endocrine Inadequacies: Hypo metabolism due to suboptimal thyroid function, hyper uricemia, and hypoglycemia.

- Chronic Infections and Infestations: Viral diseases, bacterial infection, infestations like fish tape worm.

- Miscellaneous Factors: Allergic Rhinitis, impaired sleep, nerve impairment.

\section{Classification of trigger points}

On the basis of development [1] there are three types of trigger points.

- Primary trigger points: It develops independently and not as a result of trigger point activity elsewhere.

- Secondary trigger point: It may develop in antagonist muscles and neighboring protective muscles as a result of stress and spasm. It is common for patients to experience the pain of a secondary trigger point once a primary trigger point is eliminated.

- Satellite trigger point: It can develop in the area of referred pain as a result of persistent motor unit activity in the muscle. Other classification is based on symptoms 1 production and according to this classification trigger points can be divided into 2 groups:

- Active trigger point: They are always tender, painful and symptomatic. Pain may be present at rest or on activity. On palpation these trigger points produce specific referred pain. Other clinical signs are local twitch responses (LTR) and jump sign.

- Latent trigger point: These are symptomatic and do not require treatment unless they are activated.

\section{Clinical features}

Patients with MTP in the upper trapezius muscle usually complain of neck pain, headache, muscle stiffness, restricted range of motion (ROM) of cervical joint, dizziness, sweating, vomiting and insomnia. Each muscle has its own pain referral pattern.

Pain: Patients usually complain of localized regional deep aching sensation which can vary in intensity from mild to severe.

Associated autonomic dysfunction: Disturbance in autonomic dysfunction caused by trigger point include abnormal sweating, lacrimation, excessive salivation, dizziness tinnitus etc.

Functional complaints: Functional complaints include decrease work tolerance, impaired muscle co-ordination, stiff joints, fatigue and weakness.

Neurological symptoms: Paraesthesia, numbness, blurred vision and trembling, later stages can be compounded by sleep disturbances, mood changes and stress.

Physiological effects of low level laser therapy

1. Red light affects all cell types

- Absorbed by the mitochondrial \& cytochromes present in all cells 
- $\quad$ Respiratory chain enzymes (cytochromes) within the mitochondria have been identified as the primary biostimulation chromophores (primary light-absorbing molecules).

2. Since enzymes are catalysts with the capability of processing thousands of substrate molecules, they provide amplification of initiation of a biological response with light.

3. Infrared light is more selective absorbed by specific proteins in the cell membrane \& affects permeability directly [9].

4. Analgesic effect $[15,17]$.

5. Anti-inflammatory \& anti-edematous effects $[22,23]$.

6. Increase collagen production [24].

7. Increases cell permeability [25].

8. Increases ATP production [13].

\section{Role of LLLT in MTP}

In a double blind controlled trial conducted by Gur A., et al. [6] on patients with chronic myofascial pain syndrome in neck evaluated the effects of infrared low level 904 GA-As laser therapy on clinical and quality of life. He revealed that LLLT is effective in pain relief and improvement of functional ability and quality in patients with myofascial pain syndrome.

F. Ceccherelli., et al. [27] in his study on diode laser in cervical myofascial pain confirmed that diode laser is effective and result in pain attenuation. On the contrary, Altan L., et al. [18] who investigated the effect of Ga-As laser therapy in cervical myofascial pain syndrome with a placebo controlled double blind prospective study [19]. He did not find any superiority of Ga-As laser therapy over placebo. A double blind controlled on low energy laser treatment and exercise for chronic low back pain conducted by Robin G. Klein., et al. [4] concluded that low energy laser stimulation under short term conditions does not appear to provide any advantage over exercise alone.

The study undertaken by Synder-Mackler., et al. [11] to ascertain the effects of He-Ne laser on resistance of skin resistance overlying musculoskeletal trigger point, showed significant increase in skin resistance and assumed to accompany the resolution of pathological condition. In a double blind study, repeated irradiation with a low power helium neon laser produced relief in subjects with chronic pain as concluded by J. Walker [29] whereas no statistical difference was found when subjects were treated with low output He-Ne laser therapy against placebo for chronic myofascial pain [26].

Ali Gur., et al. [14] advocated that there are differences in technology and in the devices, and differences between the geometry of the laser beam, the divergence of the beam and the system of collimation of the diode laser equipment. Because of the large number of positive reports and the innocuous nature of the therapies, further clinical evaluation of laser therapy is warranted.

Dheeraj Lamba [32,34] in his study on Comparison of Helium Neon Laser with Gallium Arsenide Laser Therapy on Pain and Functional Ability in Patients with Trigger Point (Upper Trapezius
Muscle) concluded that there was no significant difference seen in the effects of He-Ne laser application with stretching and Ga-As laser with stretching when compared to stretching alone on pain relief and functional ability. Similarly in the other study on Comparison of Two Different Photo Bio Stimulations on Pain and Functional Ability in Patients with Myofascial Trigger Points (Levator Scapulae Muscle) he concluded that significant difference in the rate of improvement was found in the group which received Ga-As laser with stretching. Since the duration over which accumulation of rate of improvement took place was small thus it could not produce any significant difference overall at the end. Hence Ga-As LLLT can be used in adjunct with stretching exercises as a cost-effective conservative treatment of MTP in levator scapulae muscle.

Hanten., et al. [10] claimed that the quality of the studies on the efficacy of these modalities was low and the supporting results reported only temporary relief from any modality. Simunovic [30] reported functional recovery and decrease of spontaneous pain with LLLT on trigger points [12].

Walker., et al. [29,31] suggested that this type of laser may effect serotonin metabolism, because of large increase in urinary excretion of 5 hydroxyindoleactic acid (5 HIAA) and better oxygenation of tissue resulting from increased local circulation hence leading to reduction of pain [6,11]. Sarac., et al. [33] found significant improvement in patients when treated with Ga-As laser with respect to parameters such as pain, functional ability and (Quality of life).

\section{Conclusion}

There has been an adequate evidence for the significant role of LLLT in musculoskeletal disorders. Therefore it is up to the physical therapist to use LLLT in his clinical setup under available evident based protocols till date for the treatment of musculoskeletal disorders particularly myofascial trigger points in and around neck. Future research is advocated on different populations to ascertain and establish LLLT as cost-effective conservative treatment of MTP which can be used in adjunct with exercises or alone.

\section{Conflict of Interest}

Nil.

\section{Source of Funding}

Self.

\section{Acknowledgement}

To Department of Physiotherapy and its staff for their valuable contribution.

\section{Bibliography}

1. Simon DG and Travell JG. "Myofascial pain and dysfunction, the trigger point manual, upper half of body, $2^{\text {nd }}$ edition". Baltimore, William and Wilkins 1 (1999).

2. Joanne Borg- Stein and David G. "Myofascial pain: A focused review". Archives of Physical Medicine and Rehabilitation 83.1 (2002): 40-47. 
3. Noramn B Rosen. "The Myofascial Pain Syndrome”. Physical Medicine and Rehabilitation Clinics of North America 4 (1993): 41-63.

4. Robert G Klein and Bjorn. "Low energy laser treatment and exercise for chronic low back pain: A double blind controlled trial". Archives of Physical Medicine and Rehabilitation 71 (1990): 34-37.

5. Leesa K and Huguenin. "Myofascial trigger points: the current evidence”. Physical Therapy in Sport 5 (2004): 2-12.

6. Gur A., et al. "Efficacy of $904 \mathrm{~nm}$ Gallium Arsenide low level laser therapy in the management of chronic myofascial pain in the neck: A double-blind and randomize-controlled trial". Lasers in Surgery and Medicine 35.3 (2004): 229-235.

7. Chang-Zern Hong. "Pathophysiology of Myofascial Trigger Point". Journal of the Formosan Medical Association 95.12 (1996): 93-104.

8. James R Friction., et al. "Myofascial pain syndrome of the head and neck: A review of clinical characteristics of 164 patients". Oral Surgery 60.6 (1985): 616-623.

9. Liisa Laakso., et al. "Factors affecting Low level laser therapy". Australian Journal of Physiotherapy 39.2 (1993): 95-99.

10. William P Hanten., et al. "Effectiveness of a home programe of Ischemic Compression by sustained stretch for treatment of Myofascial Trigger Point". Physical Therapy 80.10 (2000): 997-1003.

11. Lynn Synder-Mackler., et al. "Effect of Helium Neon Laser on Musculoskeletal Trigger Points". Physical Therapy 66.7 (1986): 1087-1090.

12. Arne Nyholm Gam., et al. "The effect of low level laser therapy on musculoskeletal pain: A Meta-analysis". Pain 52 (1993): 63-66.

13. Hakguder A., et al. "Efficacy of low level laser therapy in myofascial pain syndrome: An algometric and thermographic evaluation". Lasers in Surgery and Medicine 33 (2003): 339343.

14. Ali Gur., et al. "Efficacy of low power laser therapy and exercise on pain and functions in chronic low back pain". Lasers in Surgery and Medicine 32 (2003): 233-238.

15. Shigeo Toya., et al. "Report on a computer randomized double blind clinical trial to determine the effectiveness of the Ga-AlAs $(830 \mathrm{~nm})$ Diode laser for pain attenuation in selected pain groups". Laser Therapy 6 (1994): 143-148.

16. Leon Chaitow and Judith Walker DeLany. "Clinical Application of Neuromuscular Techniques". The upper body, Trigger Points, Churchill Livingstone 1 (2000): 65-84.
17. Roberta T Chow., et al. "A pilot study of low power laser therapy in management of chronic neck pain". Journal of Musculoskeletal Pain 12.2 (2004): 71-81.

18. Altan L., et al. "Investigation of the effect of GaAs laser therapy on cervical myofascial pain syndrome". Rheumatology International 25.1 (2003): 23-27.

19. Lale Altan., et al. "Low-Power Laser treatment for shoulder pain”. Photomedicine and Laser Surgery 23.5 (2005): 459-464.

20. O Komiyama., et al. "Posture correction as part of behavioral therapy in treatment of myofascial pain with limited opening". Journal of Oral Rehabilitation 26 (1996): 428-435.

21. Anette Kaergaard and Johan H Anderson. "Musculoskeletal disorders of the neck and shoulders in female sewing machine operators: Prevalence, incidence and prognosis". Occupational and Environmental Medicine 57 (2000): 528-534.

22. Joan McMeeken and Barry Stillman. "Perceptions of clinical efficacy of laser therapy". Australian Journal of Physiotherapy 39 (1993): 101-107.

23. Jan M Bjordal., et al. "A systematic review of low level laser therapy with location - specific doses for pain from chronic joint disorders". Australian Journal of Physiotherapy 49 (2003): 107-116.

24. John DF Allendorf., et al. Lasers in Surgery and Medicine 20 (1997): 340-345.

25. John Low and Ann Reed. "Electrotherapy Explained: Principles and Practice, $3^{\text {rd }}$ edition., Laser Therapy". Butterworth Heinemann (2000): 356-375.

26. George W. Waylonis., et al. "Chronic Myofascial Pain: Management of low output Helium Neon laser therapy". Archives of Physical Medicine and Rehabilitation 69 (1988): 1017-1020.

27. F Ceccgerelli., et al. "Diode laser in cervical myofascial pain: A double blind study versus placebo". The Clinical Journal of Pain 5 (1989): 301-304.

28. Lars Bendtsen., et al. "Qualitatively altered nociception in chronic myofascial pain". Pain 65 (1996): 259-264.

29. J Walker. "Relief from chronic pain by low power laser irradiation". Neuroscience Letters 43 (1983): 339-344.

30. Simunovic Z. "Low level laser therapy with trigger points technique: A clinical study on 243 patients". Journal of Clinical Laser Medical Surgery 14.4 (1996): 163-167.

31. Hans Jorgen Hansen and Ulla Thoroe. "Low power laser biostimulation of chronic oro-fascial pain. A double blind placebo controlled cross over study in 40 patients". Pain 43 (1990): 169-179. 
32. Dheeraj lamba. "Comparison of Helium Neon Laser with Gallium Arsenide Laser Therapy on Pain and Functional Ability in Patients with Trigger Point (Upper Trapezius Muscle)". Indian Journal of Physiotherapy and Occupational Therapy 3.2 (2009): 6-11.

33. Ali Gur., et al. "Efficacy of different therapy regimes of low power laser in painful osteoarthritis of the knee: a double blind and randomized controlled trial". Lasers in Surgery and Medicine 33 (2003): 330-338.

34. Dheeraj Lamba. "Comparison of Two Different Photo Bio Stimulations on Pain and Functional Ability in Patients with Myofascial Trigger Points (Levator Scapulae Muscle)". International Journal of Medical Research and Health Sciences 8.7 (2019): 22-29.

\section{Assets from publication with us}

- Prompt Acknowledgement after receiving the article

- Thorough Double blinded peer review

- Rapid Publication

- Issue of Publication Certificate

- High visibility of your Published work

Website: https://www.actascientific.com/

Submit Article: https://www.actascientific.com/submission.php Email us: editor@actascientific.com

Contact us: +919182824667 